\title{
The Control of Locomotor Frequency by Excitation and Inhibition
}

\author{
Wen-Chang Li and Peter R. Moult \\ School of Biology, University of St Andrews, Bute, St Andrews, Fife KY16 9TS, United Kingdom
}

Every type of neural rhythm has its own operational range of frequency. Neuronal mechanisms underlying rhythms at different frequencies, however, are poorly understood. We use a simple aquatic vertebrate, the two-day-old Xenopus tadpole, to investigate how the brainstem and spinal circuits generate swimming rhythms of different speeds. We first determined that the basic motor output pattern was not altered with varying swimming frequencies. The firing reliability of different types of rhythmic neuron involved in swimming was then analyzed. The results showed that there was a drop in the firing reliability in some inhibitory interneurons when fictive swimming slowed. We have recently established that premotor excitatory interneurons [descending interneurons (dINs)] are critical in rhythmically driving activity in the swimming circuit. Voltage-clamp recordings from dINs showed higher frequency swimming correlated with stronger background excitation and phasic inhibition, but did not correlate with phasic excitation. Two parallel mechanisms have been proposed for tadpole swimming maintenance: postinhibition rebound firing and NMDAR-dependent pacemaker firing in dINs. Rebound tests in dINs in this study showed that greater background depolarization and phasic inhibition led to faster rebound firing. Higher depolarization was previously shown to accelerate dIN pacemaker firing in the presence of NMDA. Here we show that enhancing dIN background excitation during swimming speeds up fictive swimming frequency while weakening phasic inhibition without changing background excitation slows down swimming rhythms. We conclude that both strong background excitation and phasic inhibition can promote faster tadpole swimming.

\section{Introduction}

Rhythmic neural activity is common in the vertebrate CNS (Alonso and Llinás, 1989; Buzsáki, 2002; Mann and Paulsen, 2005; Steriade, 2005; Feldman and Del Negro, 2006; Fontanini and Bower, 2006; Rivlin-Etzion et al., 2006; Grillner and Jessell, 2009). It is generally accepted that stronger excitation in the rhythm-generating network can lead to higher rhythm frequency (Brodin et al., 1985; Alford and Grillner, 1990; Cazalets et al., 1992; Talpalar and Kiehn, 2010). Weakening inhibition using pharmacological blockers, in contrast, has been shown to speed up rhythms (Dale, 1995). As the frequency of network rhythm changes, different types of neuron may be recruited (Gosgnach et al., 2006; Bhatt et al., 2007; McLean et al., 2007, 2008; Crone et al., 2009; Gabriel et al., 2011). However, it is often unclear whether neuronal recruitment is the cause or the result of network rhythm changes. It is important to reveal the basic cellular mechanisms that can translate variable excitation and inhibition levels into different network rhythm frequencies in an identified circuit.

We use a well established vertebrate model, the Xenopus tadpole, to study how its swimming frequency is controlled. The tadpole swimming circuit contains four identified types of rhyth-

Received Dec. 19, 2011; revised March 16, 2012; accepted March 19, 2012.

Author contributions: W.-C.L. designed research; W.-C.L. and P.R.M. performed research; W.-C.L. analyzed data; W.-C.L. and P.R.M. wrote the paper.

This work was supported by the Royal Society and Wellcome Trust. We thank Glen Cottrell, William Heitler, and Nicola Porter for commenting on this manuscript.

Correspondence should be addressed to Wen-Chang Li, School of Biology, University of St Andrews, Bute, West Burn Lane, St Andrews, Fife KY16 9TS, UK. E-mail: w121@st-andrews.ac.uk.

DOI:10.1523/JNEUROSCI.6289-11.2012

Copyright $\odot 2012$ the authors $\quad 0270-6474 / 12 / 326220-11 \$ 15.00 / 0$ mic neuron located throughout the spinal cord and caudal hindbrain. Their transmitter phenotypes have been established using immunocytochemistry and/or pharmacology. The synaptic connections between these neurons have been revealed using simultaneous recordings (Roberts et al., 2010). Tadpole swimming rhythms can be generated through either rebound firing following rhythmic inhibition (Soffe et al., 2009) or conditional pacemaker firing following NMDAR activation in the premotor excitatory interneurons ( $\mathrm{Li}$ et al., 2010), the descending interneurons with ipsilateral axons (dINs). Both mechanisms require the depolarization produced by NMDARs but the former also depends on phasic inhibition (Li, 2011).

We find that high-frequency swimming is correlated to both strong background excitation and strong phasic inhibition. Recordings from dINs show that both can lead to faster dIN rebound firing and faster swimming rhythms.

\section{Materials and Methods}

Human chorionic gonadotropin was regularly injected in pairs of adult male and female Xenopus to induce mating and embryos' incubation temperatures were then changed to stagger their developmental rates. All experiment procedures were approved by the local Animal Welfare Ethics committee and complied with UK Home Office regulations. Following brief anaesthetization (0.1\% MS222, 3-aminobenzoic acid ester; Sigma), the tadpole (stage 37/38) dorsal fin was cut open to allow immobilization using $\alpha$-bungarotoxin (12.5 $\mu \mathrm{M}$; Tocris Cookson) and the animal was pinned onto a sylgard stage for dissections. Dissections and recordings were performed in saline containing the following (in $\mathrm{mm}$ ): $\mathrm{NaCl} 115, \mathrm{KCl} 3, \mathrm{CaCl}_{2} 2, \mathrm{NaHCO}_{3} 2.4, \mathrm{MgCl}_{2} 1$, HEPES 10, with $\mathrm{pH}$ adjusted to 7.4 with $\mathrm{NaOH}$. $\mathrm{NaCl}$ was replaced with equimolar sodium methyl sulfate to change IPSP reversal in some experiments. The tadpole 

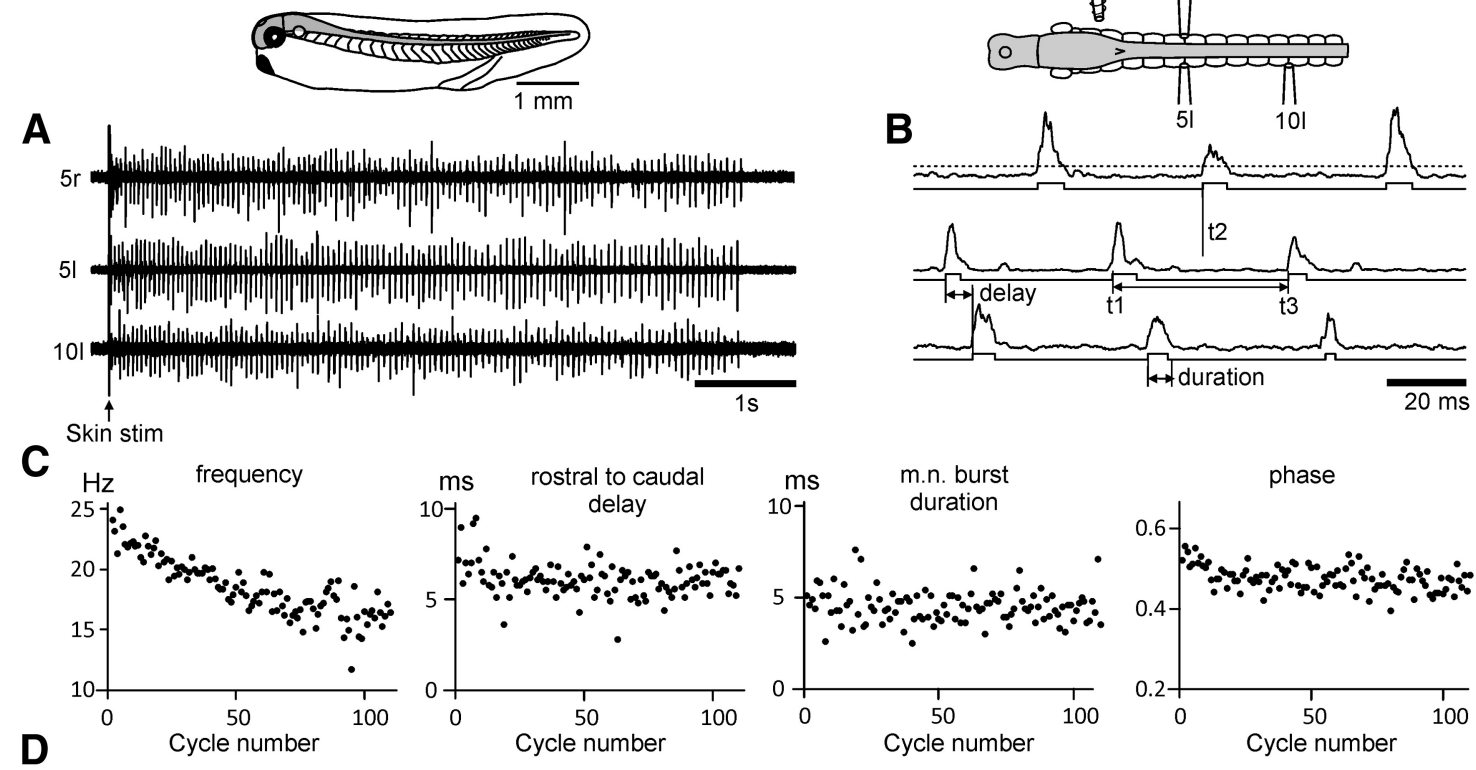

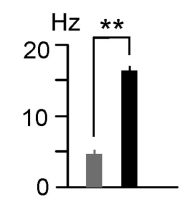

frequency

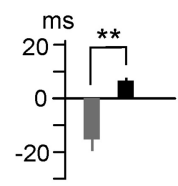

rostral to caudal delay
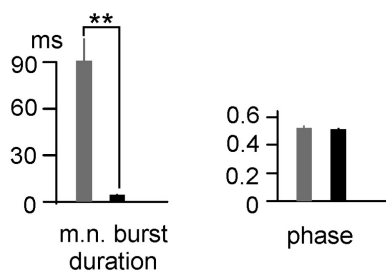
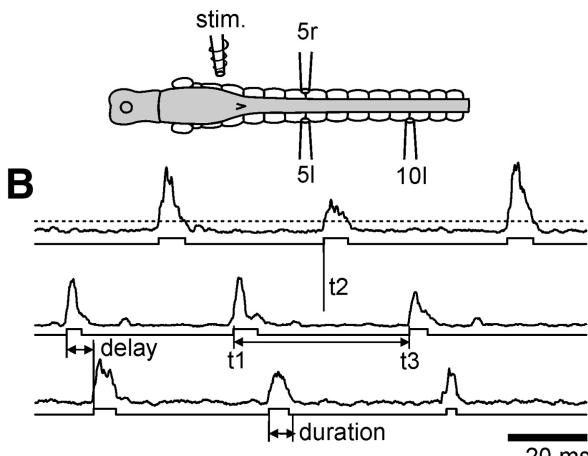

$20 \mathrm{~ms}$

Figure 1. Tadpole motor output pattern remains the same when swimming frequency changes. $\boldsymbol{A}$, Simultaneous recordings of a swimming episode from three $\mathrm{m}$.n. [fifth left (5l), fifth right ( $5 \mathrm{r}$ ), 10th left (10l)]. $\boldsymbol{B}$, Expanded and rectified swimming records to show how measurements are made to evaluate motor outputs. Events (steps on straight lines below m.n. traces) are triggered by threshold (dotted line) crossing. Phase $=(\mathrm{t} 2-\mathrm{t} 1) /(\mathrm{t} 3-\mathrm{t} 1)$. $\boldsymbol{C}$, Time series measurements of swimming frequency, rostral-to-caudal delay, m.n. burst duration, and phase from the records in $\boldsymbol{A}$. Abscissa is cycle number from the start of the episode. $\boldsymbol{D}$, Comparing frequency, rostral-to-caudal delay, m.n. burst duration, and phase in swimming to those in struggling in six tadpoles. **Significance level at $p<0.01$ ( $t$ test). Top left diagram shows a stage 37/38 tadpole. Top right diagram illustrates electrodes and the experimental setup. Stim., Stimulation electrode.

hindbrain and rostral spinal cord were opened up dorsally using a fine tungsten needle. Further small cuts were made to remove the ependymal cells lining the central canal to expose neuronal cell bodies to make access for whole-cell recording electrodes.

Standard whole-cell recordings were made in either current-clamp or voltage-clamp mode. Patch pipettes were filled with $0.1 \%$ neurobiotin (Vector Labs) in the intracellular solution (concentrations in $\mathrm{mm:}$ K-gluconate 100, $\mathrm{MgCl}_{2}$ 2, EGTA 10, HEPES 10, $\mathrm{Na}_{2}$ ATP 3, NaGTP 0.5 adjusted to $\mathrm{pH} 7.3$ with $\mathrm{KOH}$ ) with DC resistances ranging from 10 to 20 $\mathrm{M} \Omega$. In some experiments, K-gluconate was replaced with equimolar $\mathrm{KCl}$ to change the IPSP reversal. Some whole-cell recordings were from data used in previous papers by $\mathrm{Li}$ and colleagues where whole-cell recordings were made with Axon-2B amplifier, CED 1401 plus digitizer, and Signal software (Cambridge Electronic Design). Other whole-cell recordings were made using Axon Multiclamp 700B, digitized with Digidata 1440A or Power $1401 \mathrm{mkII}$, and sampled with pClamp 10 software (Molecular Devices) or Signal (version 4). Fictive swimming was evoked by applying single or repetitive $1 \mathrm{~ms}$ current pulses to the skin of immobilized tadpoles. We used the term swimming instead of fictive swimming for simplicity in the text. Motor nerve (m.n.) recordings were made by placing glass suction electrodes ( $\sim 50 \mu \mathrm{m}$ in tip diameter) on the segmented swimming muscle clefts in the body trunk (normally between the fifth and 10th muscle segments) where motor neuron axons are shallow and therefore easy to record a good signal. Final identification of neurons was based on their anatomy as revealed by neurobiotin staining after recording. To evoke rebound firing in dINs, a suction electrode was placed on one side of spinal cord surface to apply electrical stimuli to excite commissural inhibitory interneurons and dINs were recorded on the opposite side. In these cases, excitatory synaptic transmission from sensory interneurons with commissural axons was blocked using NBQX (AMPA receptors, 2,3- dihydroxy-6-nitro-7-sulfamoylbenzo-[f]quinoxaline), D-AP5 NMDAR, D-(-)-2-amino-5-phosphonopentanoic acid; Tocris Cookson) and $\mathrm{DH} \beta \mathrm{e}$ (nicotinic receptors, dihydro- $\beta$-erythroidine; Research Biochemicals International).

Data processing and analyses were performed using Dataview (v6.1, courtesy of Dr W. J. Heitler, University of St Andrews, Fife, UK) and Excel. For measuring firing reliability of each type of rhythmic neuron, the number of spikes on each swimming cycle was documented in order for the first 80 swimming cycles. Firing reliability was calculated for each cycle as the total number of spikes divided by the number of neurons measured. To measure both EPSC and IPSC, dIN membrane potential was clamped around either EPSC $(\sim 5 \mathrm{mV})$ or IPSC reversal $(\sim-55 \mathrm{mV})$ levels. They were measured, normalized to the average of the first three cycles in individual recordings, and then averaged for each swimming cycle in each type of rhythmic neuron. dIN rebound firing time was measured as the delay from the start of inhibition (IPSP start or current injection onset) to dIN spike peak. Equivalent dIN rebound firing frequency was calculated as if this time accounted for a half swimming cycle.

Statistical analyses were performed using PASW statistics 18 (IBM SPSS). Normality tests were performed routinely on datasets. Means were given with standard errors (mean \pm SEM) and compared using $t$ tests for normally distributed data. In other cases, Wilcoxon signed rank test was used. Pearson correlation (two-tailed) was performed between neuronal firing reliability, synaptic current amplitudes, and swimming frequencies. To evaluate the effect of injecting direct currents (DC) into individual rhythmic neurons on the frequency of swimming, the average frequency of 5-15 swimming cycles was measured during and immediately before current injection. Because some current injections lasted for long periods over which an endogenous gradual decrease in swimming frequency occurred, the frequency of swimming cycles after current in- 
jection were not measured. Trials from many neurons (1-4 trials from each neuron) were pooled together for comparison and statistical significance tested using paired $t$ tests.

\section{Results}

Motor output pattern does not change when swimming frequency changes

First, we confirmed that the swimming motor output pattern remained the same when swimming frequency changed. Simultaneous m.n. recordings were made from three chosen longitudinal positions (fifth left, fifth right, and 10th left swimming muscle clefts) in six tadpoles. The m.n. burst parameters measured were as follows: frequency, duration, left-right coordination (phase), and rostral-caudal delay (Fig. 1 $A, B$ ). Swimming was evoked by one or two brief electrical pulses applied on tadpole trunk skin. During swimming $(16.4 \pm 0.7 \mathrm{~Hz})$, m.n. bursts on each cycle are brief $(4.2 \pm 0.5 \mathrm{~ms})$, there was a rostral-to-caudal delay $(6.4 \pm 1 \mathrm{~ms})$, and regular left-right alternation (phase: $0.51 \pm$ $0.01,970$ cycles analyzed). When time series plots of swimming frequency, burst duration, phase, and rostral-to-caudal delay were drawn, the measurements all showed little or gradual change (Fig. 1C). We also induced fictive struggling in these six tadpoles, which behaviorally would produce backward thrusts for tadpoles (Soffe, 1991), to illustrate how motor output pattern could differ in another mode of motor output. Compared with swimming, the m.n. burst parameters for fictive struggling were very different (frequency: $4.7 \pm 0.6 \mathrm{~Hz}$, burst duration: $92.6 \pm 13.6 \mathrm{~ms}$, rostral-to-caudal delay: $-15.3 \pm 4.3 \mathrm{~ms}$, all $p<0.01)$ except for phase $(0.52 \pm 0.02, n=74$ cycles analyzed; Fig. 1D). This was in line with previous observations that the motor output patterns remained unchanged with swimming frequency (Soffe, 1991; Roberts et al., 2010).

\section{Firing reliability of neurons}

rhythmically active in swimming

The frequency of swimming drops most prominently within the first few seconds after its initiation and we analyzed the firing reliability of rhythmic neurons in current-clamp mode within the first 80 cycles ( $\sim 5 \mathrm{~s}$ from the beginning of each episode). The four types of rhythmic neurons in the tadpole swimming circuit (Roberts et al., 2010) are dINs (Fig. 2A), ipsilateral inhibitory interneurons [ascending interneurons (aINs); Fig. 2B], motoneurons (Fig. 2C), and commissural inhibitory interneurons (cINs; Fig. 2D). The firing reliability was first calculated as the average number of spikes for every swimming cycle in each type of neuron. This was used for time series plots (Fig.

A m.n. same side as the m.n. in $\boldsymbol{D}$.

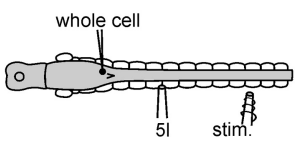

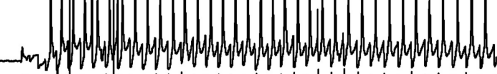

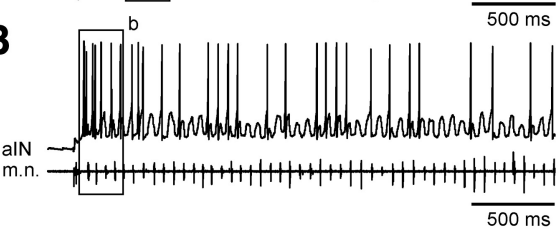

a
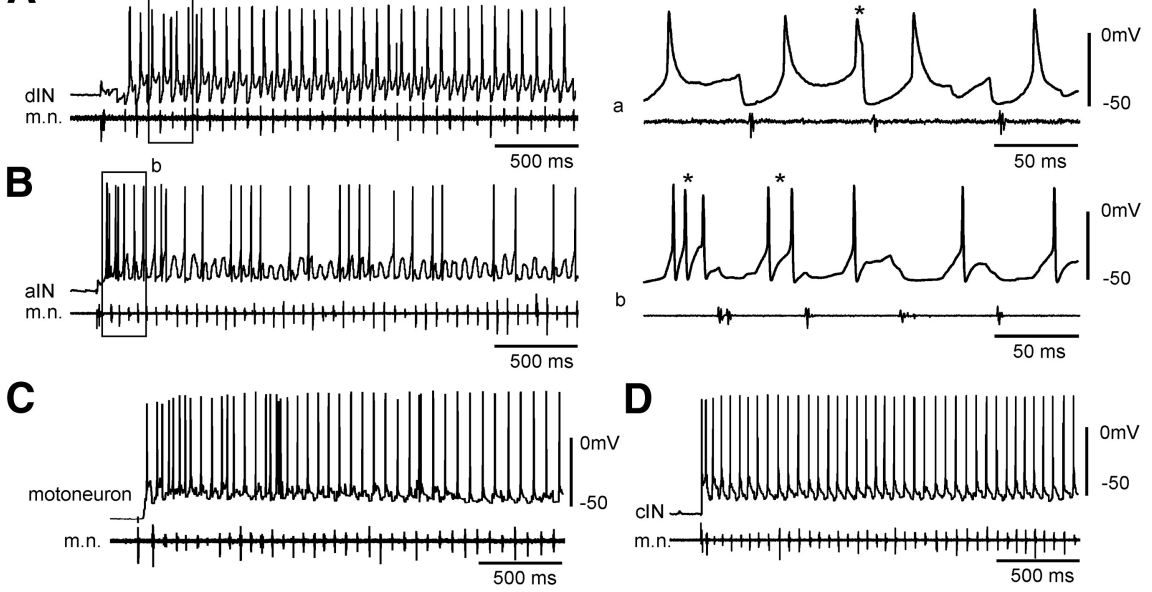

Figure 2. The activity of rhythmic neurons in tadpole swimming. $A$, The firing of a dIN at the beginning of a swimming episode. $\boldsymbol{B}-\boldsymbol{D}$. The firing of an aIN $(\boldsymbol{B})$, a motoneuron $(\boldsymbol{C})$, and a $\mathrm{CIN}(\boldsymbol{D})$ at the start of swimming. $\boldsymbol{a}, \boldsymbol{b}$, Records in the boxed regions in $\boldsymbol{A}$ and $\boldsymbol{B}$ on a smaller time scale to show multiple firing in single swimming cycles $\left({ }^{*}\right)$. cIN was recorded from the
A

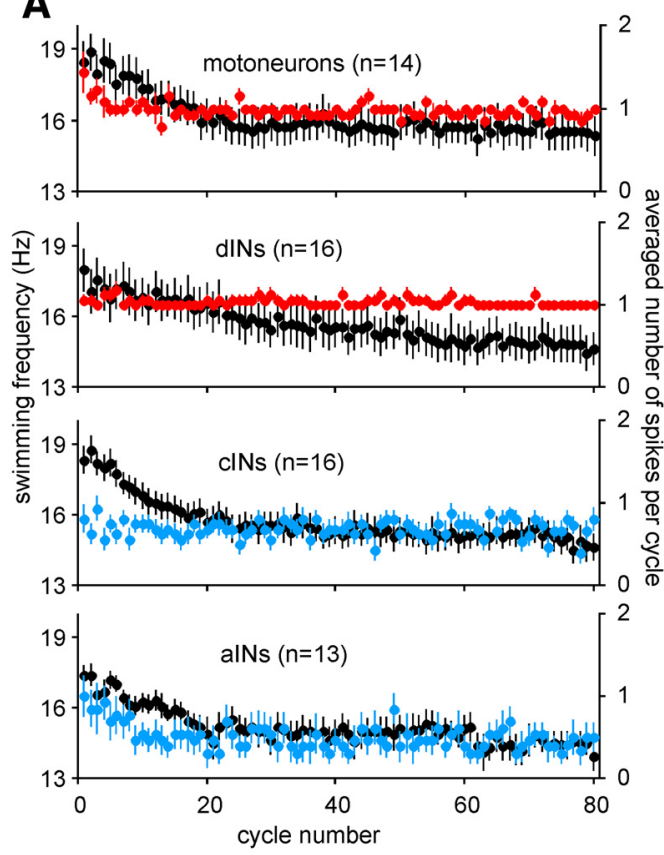

B motoneurons

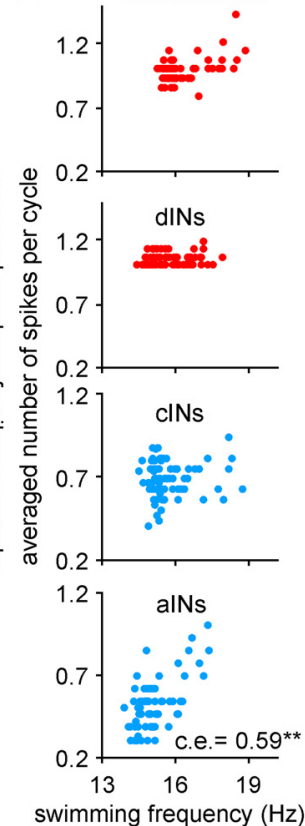

Figure 3. The correlation of firing reliability with swimming frequency in the four types of neuron. $\boldsymbol{A}$, Swimming frequency (black symbols, left ordinate) and neuronal firing reliability (red and blue symbols, right ordinate) in the first 80 cycles following swimming initiation. A reliability of 1 means the neuron fires an average of one spike on the chosen swimming cycle. Vertical bars are SEs. $\boldsymbol{B}$, Swimming frequencies plotted against neuronal firing reliability measurements using the same data in $\boldsymbol{A}$. c.e., Correlation coefficient. **Significance level at $p<0.01$.

$3 A)$ and correlation analysis. An overall firing reliability was also given for each neuron group by averaging cycle-by-cycle measurements over a stable period of time. dINs firing reliability overall remained high $(1.03 \pm 0.04)$ in all 80 cycles $(n=16)$. aINs fired mostly unreliably $(n=13)$. Their reliability dropped fast in the first 15 cycles or so and remained largely stable at $0.46 \pm 0.12$ afterward. Motoneuron firing reliability dropped quickly within the first few cycles and then stayed at $0.97 \pm 0.06$ spikes per cycle 


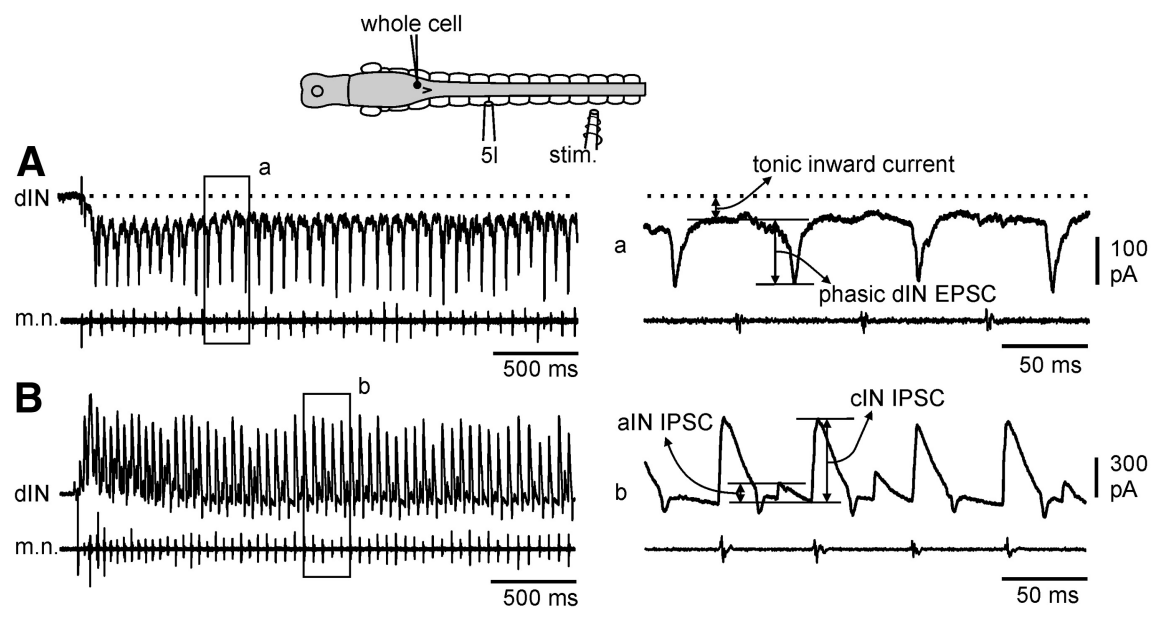

Figure 4. Synaptic currents in dINs during swimming. $A$, Tonic inward currents and phasic EPSCs a dIN receives during swimming. dIN membrane potential was clamped around the IPSC reversal $(-55 \mathrm{mV})$. Tonic inward current is the difference between clamping current at rest (dotted line) and current at the flat midpoint between two fast phasic dIN EPSCs. Phasic dIN EPSCs are measured as the difference between peak inward current and the same flat midpoint level. B, IPSCs in a dIN during swimming. aIN IPSCs and cIN IPSCs are measured as the difference between peak outward currents and the current level just before IN IPSCs in each cycle. $\boldsymbol{a}, \boldsymbol{b}$, Boxed regions from $\boldsymbol{A}$ and $\boldsymbol{B}$ displayed with a smaller time scale.
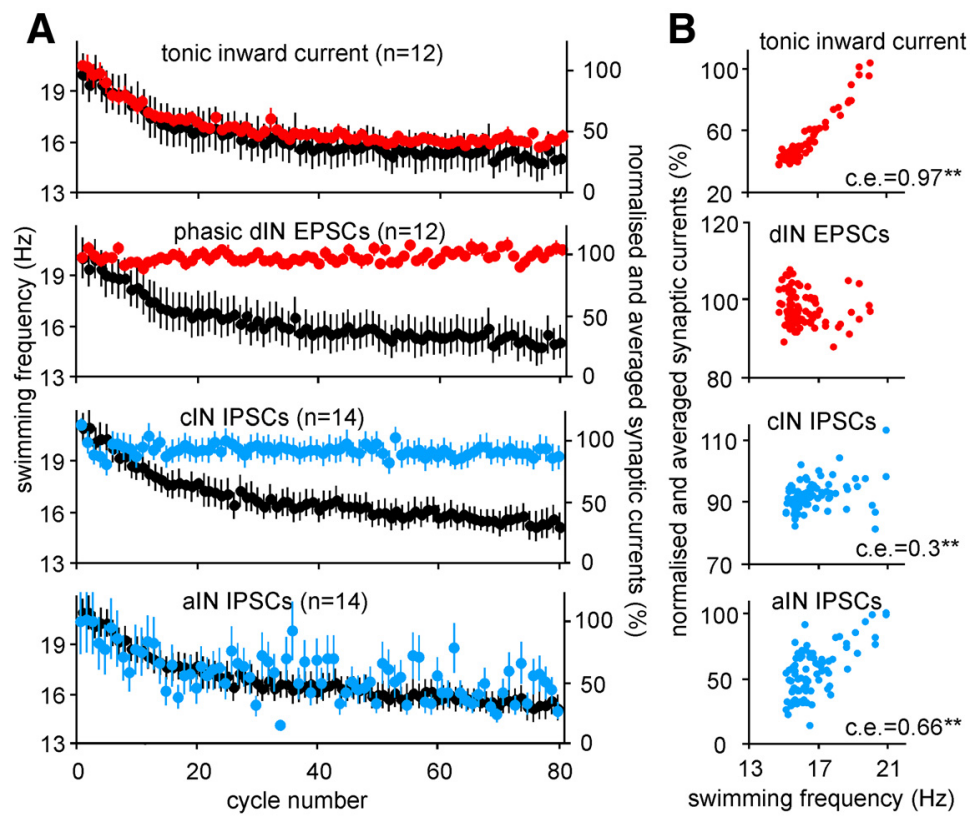

Figure 5. The correlation between swimming frequency and the amplitude of different synaptic currents in dINs. A, Swimming frequency (black symbols, left ordinate) and different synaptic currents (red and blue symbols, right ordinate) in dINs in the first 80 cycles following swimming initiation. Current amplitude is normalized to the average of the first three cycles for each type of current in individual recordings and then averaged. Vertical bars are SES. B, Swimming frequencies plotted against normalized and averaged synaptic currents using the same data in $\boldsymbol{A}$. c.e., Correlation coefficient. ${ }^{*}$ Significance level at $p<0.01$.

$(n=14)$. cIN firing reliability remained fairly stable at $0.79 \pm$ 0.12 spikes per cycle $(n=16)$. We analyzed the cycle-by-cycle reliability of neuronal firing in relation to swimming frequency. The swimming frequency was correlated with aIN firing reliability (two-tailed Pearson coefficient: $0.585, p<0.0001$ ), but not with $\mathrm{dIN}$ or cIN firing reliability $(p>0.05)$. Extra motoneuron firing took place mostly within the first three cycles $(1.43,1.14$, and 1.21 spikes per cycle). Motoneuron firing reliability was not correlated with swimming frequency from the fourth cycle onwards (Fig. 3B).

\section{Synaptic currents in dINs} during swimming

We have recently shown that dINs are the driver neurons for tadpole swimming and they drive the activity of other neurons in a rhythmic manner (Soffe et al., 2009; Li et al., 2010). It is important to reveal changes in the synaptic currents that underlie dIN firing during swimming. During swimming, all rhythmic neurons receive phasic inhibition from aINs and cINs and phasic excitation from dINs on top of a tonic background depolarization (Roberts et al., 2010). We recorded dINs in voltageclamp mode (Fig. 4) so we could correlate the changes in dIN synaptic drives (outputs from rhythmic neurons during swimming and sensory inputs at the episode start) with swimming frequency in the first 80 swimming cycles (Fig. 5). Synaptic current amplitude was normalized to the average currents of the first three cycles in individual recordings and then the normalized amplitude was averaged cycle-by-cycle using data from many recordings. Both sensory pathway neurons and dINs themselves can activate NMDARs on dINs (Roberts et al., 2010), producing tonic inward current giving rise to a background excitation. The amplitude of tonic inward current correlated with swimming frequency $(n=12 \mathrm{dINs}$, coefficient: $0.963, p<0.0001$ ). There was no correlation between fast phasic EPSCs produced by dINs and swimming frequency ( $n=12 \mathrm{dINs}, p>0.05)$, which is in agreement with stable dIN firing reliability. The correlation between the decrease in IPSC amplitude produced by cINs and swimming frequency was less obvious, but was significant ( $n=14 \mathrm{dINs}$, coefficient: $0.323, p=0.003$ ). The amplitude of IPSCs produced by aINs also correlated with swimming frequency $(n=14$ dINs, coefficient: $0.658, p<0.0001)$. This agrees with the observation above that aIN firing reliability dropped with swimming frequency throughout the first 80 cycles.

These results reveal that tonic inward currents and phasic IPSCs (but not phasic EPSCs) in dINs correlate with swimming frequency. We next injected currents into dINs at rest to simulate these synaptic currents to see how they could affect dIN firing. cINs were also stimulated electrically by placing a suction electrode on the opposite side of the spinal cord to produce phasic inhibition.

Both strong background depolarization and phasic inhibition can speed up dIN rebound firing

dINs typically only fire a single action potential at the onset of fast depolarization but can fire again following either phasic inhibition (rebound firing) or repetitively when their NMDARs are 
activated (pacemaker firing) (Li, 2011). Pacemaker firing does not involve phasic inhibition and the relationship between depolarization and dIN pacemaker firing frequency has been revealed recently (Li et al., 2010). Although dIN firing frequency is restrained within the normal swimming frequency range, larger depolarization induces faster NMDAR-dependent dIN firing. We further investigated the factors that could affect dIN rebound firing speed in this study.

To test the effect of background depolarization on dIN rebound firing frequency, dIN firing was evoked by superimposing a fixed negative ramp current (decreasing from a set level to 0 over $25 \mathrm{~ms}$ ) on top of positive step currents of different sizes (duration: $0.5-1 \mathrm{~s}$ ). We found that higher depolarization $(-3 \sim-35 \mathrm{mV})$ produced faster rebound firing $(n=6$, equivalent to $0.344 \pm$ $0.07 \mathrm{~Hz} / \mathrm{mV}$ in linear regression coefficients; Fig. $6 B$, red symbols). A similar relationship between background depolarization and dIN rebound firing frequency was observed when IPSPs were evoked by electrically stimulating the opposite side of the spinal cord to induce dIN rebound firing $(6 \mu \mathrm{M}$ NBQX, $2 \mu \mathrm{M}$ DH $\beta$ e, and $60 \mu \mathrm{M}$ D-AP5 were used to block excitatory neurotransmission, $n=6$, equivalent to $0.51 \pm 0.08$ $\mathrm{Hz} / \mathrm{mV}$, regression coefficients compared, $p>0.05, t$ test; Fig. $6 A, B$, black symbols).

To examine the effect of phasic cIN inhibition on dIN rebound firing frequency, negative ramp currents with various strengths (25 ms in duration) were superimposed on a fixed suprathreshold positive step current (0.5-1 s in duration). When the inhibition trough was below $-43.2 \pm 3 \mathrm{mV}(n=7 \mathrm{dINs})$, there was no clear correlation between inhibition size and dIN rebound firing frequency. However, when inhibition was smaller, weak inhibition led to slower dIN rebound firing $(n=13$ dINs; Fig. 6C,E, red symbols). Fitting the linear part of scatter plots revealed a coefficient of $-0.24 \pm 0.07$ $\mathrm{Hz} / \mathrm{mV}$ ( $n=7$ dINs). When the opposite side of the spinal cord was stimulated to evoke rebound firing, stimulus intensity was altered to produce various size IPSPs $(n=$ $6 \mathrm{dINs}$ ). In five of six dINs, larger IPSPs produced faster rebound firing. Linear fitting of the scatter plots gave an average coefficient of $-0.3 \pm 0.07 \mathrm{~Hz} / \mathrm{mV}$ (Fig. $6 D, E$, black symbols).

Since aIN firing and IPSCs are correlated with swimming frequency, we examined the effects of aIN inhibition on dIN rebound firing frequency. aIN inhibition precedes cIN inhibition in the same swimming cycle (Fig. $4 b$ ). We therefore added a second negative ramp current injection $20 \mathrm{~ms}$ (a typical gap between aIN and cIN IPSCs in a swimming cycle) before the main ramp current to simulate aIN inhibition (Fig. 6F). Results showed that, when the main inhibition was large (average trough was $\sim-50 \mathrm{mV}, n=5 \mathrm{dINs}$ ), adding aIN inhibition did not
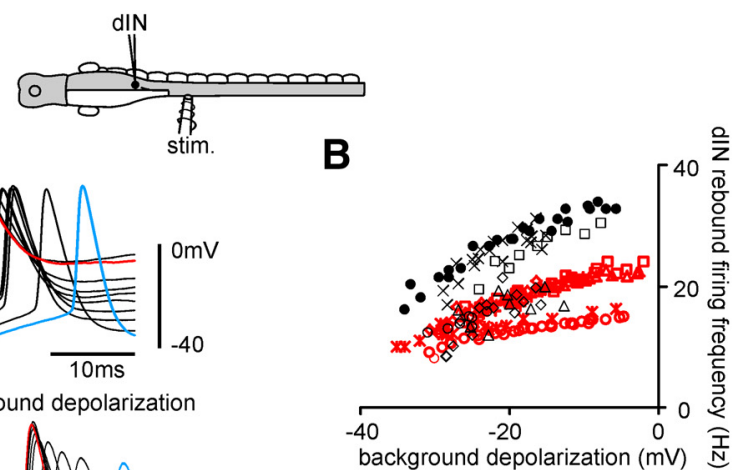

6. The effect of varying background depolarization and phasic inhibition on dIN rebound firing speed. $\boldsymbol{A}$, dIN rebound speds up when background depolarization is increased by DC current injection. IPSPs are evoked by stimulating the cord on the opposite side $\left(^{*}\right)$. The time between the start of IPSP and spike peak is treated as an equivalent half swimming-cycle period. This period is used to calculate equivalent dIN rebound firing frequencies. $\boldsymbol{B}$, Scatter plot of background depolarization levels drawn (n) dIN rebound firing frequencies. $\boldsymbol{C}$, dIN rebound firing is delayed when the $\mathrm{IN}$-like inhibition produced by ramp ent injection (I) is weak. Inset shows a full current injection protocol and dIN response. $\boldsymbol{D}$, Similar to $\boldsymbol{C}$ but cIN inhibition is current and positive step current fixed and the early ramp current (I) stepped to produce alN-like inhibition. $G$, Scatter plot summarizing tests in $\boldsymbol{F}$ with aIN-like inhibition trough drawn against equivalent dIN rebound firing frequencies. Red symbols are tests when the main inhibition is small (left ordinate) and orange symbols are trials when the main inhibition is large (right ordinate). Different symbols are from different dINs and filled symbols in $\boldsymbol{B}, \boldsymbol{E}$, and $\boldsymbol{G}$ are for the records shown in $\boldsymbol{A}, \boldsymbol{C}, \boldsymbol{D}$, and $\boldsymbol{F}$. The xperiments and black symbols are experiments when inhibition is evoked by spinal cord stimulation. Top diagram shows the hindbrain is removed on the stimulated side.

significantly affect dIN rebound firing speed (equivalent to $-0.008 \pm 0.005 \mathrm{~Hz} / \mathrm{mV}$; Fig. $6 G$, orange symbols). When the main inhibition was small (average trough was $\sim-25 \mathrm{mV}, n=5$ dINs), early inhibition tended to speed up dIN firing (equivalent to $-0.08 \pm 0.02 \mathrm{~Hz} / \mathrm{mV}, p<0.05, t$ test, compared with large main inhibition trials; Fig. $6 F, G$, red symbols). This suggests that aIN inhibition can assist the main cIN inhibition during swimming to speed up dIN rebound firing.

\section{Altering background excitation in dINs affects swimming frequency}

We next altered background depolarization in dINs during swimming to see whether this could affect swimming frequency. There is extensive electrical coupling between dINs ( $\mathrm{Li}$ et al., 2009). DC injected into one dIN can spread into coupled dINs 

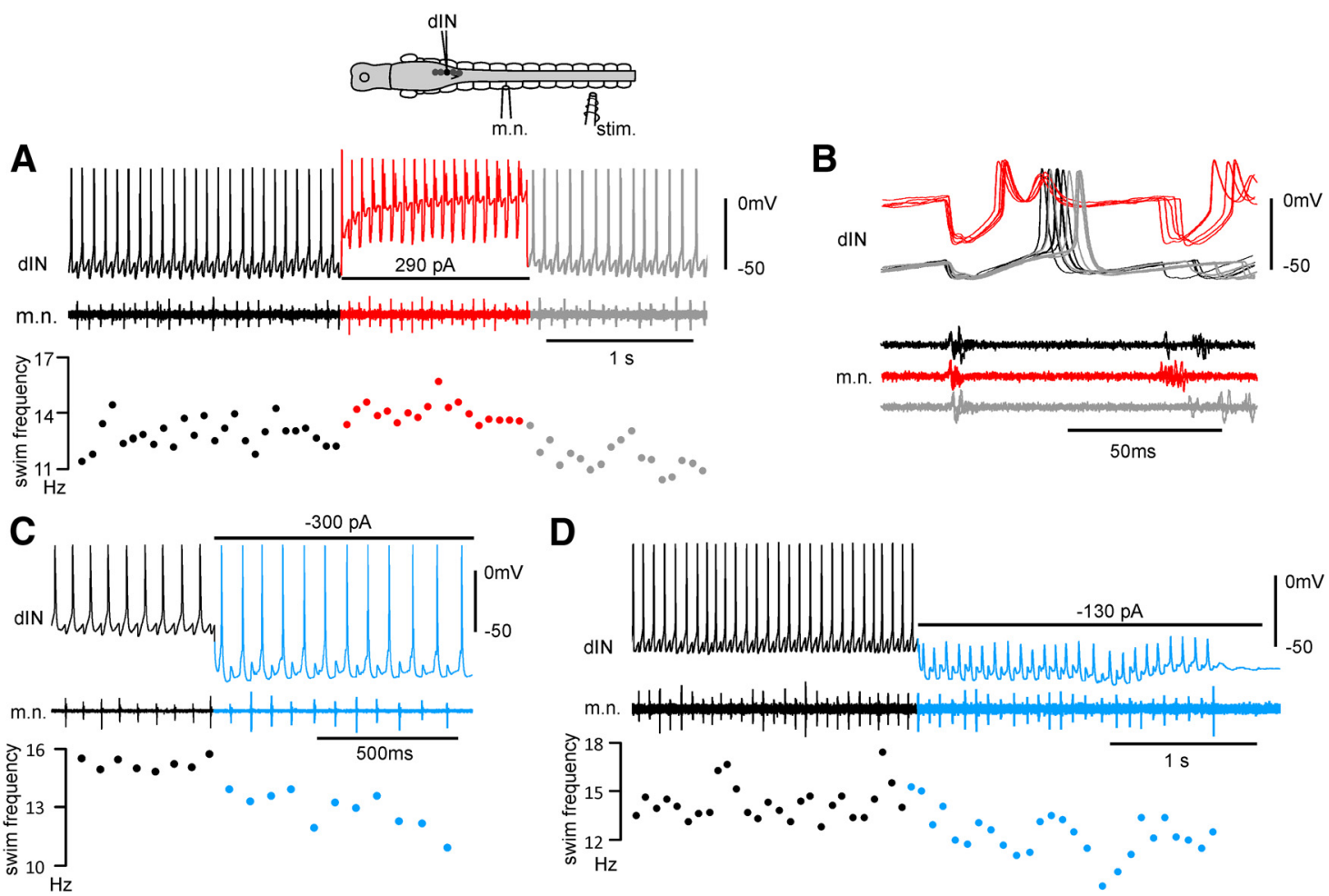

Figure 7. Changing dIN background depolarization during swimming can alter swimming frequency. $A$, Injecting $290 \mathrm{pADC}$ current in a dIN instantaneously increases swimming frequency from $12.9 \pm 0.1$ to $14 \pm 0.1 \mathrm{~Hz}$ (27 cycles before and 18 cycles during DC in this trial were compared, $p<0.0001, t$ test), $B$, Overlapped five cycles of swimming and dIN activity before (black), during (red), and after (gray) DC injection. C, Negative DC injection in a dIN decreases swimming frequency from $15.4 \pm 0.1 \mathrm{~Hz}$ before $D C$ to $12.8 \pm 0.2 \mathrm{~Hz}$ during $D C$ ( 11 cycles each were compared in this trial, $p<0.0001$, $t$ test) without inhibiting the firing in the recorded dIN. $D$, Negative $D$ C current in another dIN leads to failure of dIN firing, lower swimming frequencies (from control of $14.3 \pm$ $0.2 \mathrm{~Hz}$ to $12 \pm 0.2 \mathrm{~Hz}$ during $D C, 37$ cycles before and 22 cycle during $D C$ in this trial were compared, $p<0.0001, t$ test) and likely ending of swimming. Time series frequency measurements of swimming are given below the records in $\boldsymbol{A}, \boldsymbol{C}$, and $\boldsymbol{D}$.

and affect their firing as a subgroup, which in turn may alter swimming. Among 28 dINs, positive DC injections into dINs in the caudal hindbrain area could, in many cases, instantaneously increase swimming frequency (Fig. $7 A, B$ ). Large negative DC injection into these dINs often slowed down swimming rhythms instantly (Fig. 7C) or occasionally appeared to have stopped swimming altogether (Fig. 7D). We measured the average swimming frequency for cycles during DC injections and for a similar number of cycles immediately before DC injections in these dINs (5-15 cycles depending on DC duration in each trial, 2-4 DC trials measured in each dIN; Fig. 8A). Results showed that when positive DC was injected, swimming frequency increased from $14.4 \pm 0.23$ to $15.2 \pm 0.25 \mathrm{~Hz}(n=95$ trials, $p<0.001$, paired $t$ test). Many trials showed $>5 \%$ increase in swimming frequency $(n=46)$. When negative DC was injected, swimming frequency dropped from $14.8 \pm 0.23$ to $13.7 \pm 0.23 \mathrm{~Hz}(n=108$ trials, $p<$ 0.001 , paired $t$ test) with 64 trials showing $>5 \%$ decrease (Fig. $8 C, D)$. In contrast, when DC was injected into non-dINs $(20$ motoneurons, 12 cINs, 3 aINs), a change in swimming frequency was not observed (1-3 trials measured from each neuron, 75 positive DC trials, 64 negative DC trials, $p>0.1$ for both types of DC injection, paired $t$ test; Fig. $8 B-D$ ). This was true when the inhibitory interneurons were analyzed separately or when pooled together with the motoneurons.

\section{Specifically weakening inhibition in dINs during swimming slows down swimming}

Based on dIN rebound tests above, weakening inhibition during swimming should lead to a drop in swimming frequency. Applying a low concentration of strychnine $(<0.2 \mu \mathrm{M})$, however, in- creased fictive swimming frequency (Dale, 1995). We saw similar results when tadpoles were briefly bathed with $0.07-0.25 \mu \mathrm{M}$ strychnine. Low concentrations of strychnine did not change the resting membrane potential, input resistance, or firing properties of neurons $(p>0.05$ in all cases, paired $t$ test, spike height is the difference between spike peak and afterhyperpolarization trough; Table 1). Whole-cell recordings revealed that strychnine application enhanced background depolarization in dINs and other types of neurons when $\mathrm{Mg}^{2+}$ was present in the saline (control: $14.8 \pm 1.2 \mathrm{mV}$; strychnine: $19.7 \pm 1.6 \mathrm{mV}$, paired $t$ test, $n=5$, $p<0.01$; Fig. $9 A$ ) or omitted (control: $25.5 \pm 1 \mathrm{mV}$; strychnine: $32.7 \pm 1.7 \mathrm{mV}$, paired $t$ test, $n=7, p<0.01$; Fig. $9 B$ ). Since changing membrane potentials can affect $\mathrm{Mg}^{2+}$ block of NMDARs (Mayer et al., 1984) and $\mathrm{Mg}^{2+}$ is also required in dIN NMDAR-dependent pacemaker firing (Li et al., 2010), we omitted $\mathrm{Mg}^{2+}$ from the saline to simplify the experiments. Tadpoles can produce fictive swimming at frequencies within the normal swimming range in $\mathrm{Mg}^{2+}$ free saline (Roberts et al., 2010).

We reduced extracellular $\mathrm{Cl}^{-}$to $25 \mathrm{~mm}$ to weaken glycinergic IPSPs. This was done in $\mathrm{Mg}^{2+}$ free saline in six tadpoles, crosssectioned at seventh-eighth rhombomere border in the brainstem to cutoff any upper-brain inputs to the swimming circuit (Roberts, 1978; Perrins et al., 2002). Whole-cell recordings showed that saline with $25 \mathrm{~mm} \mathrm{Cl}^{-}$did not change the background depolarization level in rhythmic neurons during swimming (control: $25.4 \pm 1 \mathrm{mV}, 25 \mathrm{mM} \mathrm{Cl}^{-}$: $25.2 \pm 1.2 \mathrm{mV}, p<$ 0.05 , paired $t$ test, $n=6$; Fig. $9 C$ ). Swimming frequency was lowered to $77.7 \pm 0.5 \%$ of the control values by saline containing $25 \mathrm{mM} \mathrm{Cl}^{-}(p<0.001$, Wilcoxon signed rank test; Fig. 10A). When there was $1 \mathrm{mM} \mathrm{Mg}^{2+}$ in the saline, a similar drop in 
swimming frequency to $88.1 \pm 0.5 \%$ of control was seen in five tadpoles $(p<$ 0.001, Wilcoxon signed rank test; Fig. $10 \mathrm{~B}, \mathrm{C})$, suggesting that in normal saline, stronger inhibition can also promote faster swimming.

We then looked for ways to specifically weaken phasic inhibition in just dINs in 0 $\mathrm{Mg}^{2+}$ saline. First, we tried to increase intracellular $\mathrm{Cl}^{-}$. Recording neurons with single electrodes filled with $100 \mathrm{~mm} \mathrm{KCl}$ in most cases made IPSPs excitatory and evoked firing. To avoid this, two electrodes (one with standard pipette solution and the other with $100 \mathrm{mM} \mathrm{KCl}$ ) were used to record the same dIN simultaneously. The level of $\mathrm{Cl}^{-}$diffusion from the 100 $\mathrm{mm} \mathrm{KCl}$ electrode can be adjusted by slightly withdrawing the electrode away from dIN soma for a few micrometers. Such a small displacement of electrode often resulted in the formation of a fine membranous tube between the electrode tip and soma, presumably restricting the exchange of ions between the electrolyte and the cytoplasm. IPSPs could either be reduced in amplitude or reversed depending on how much the $100 \mathrm{~mm} \mathrm{KCl}$ electrode was withdrawn. Weakening IPSPs in this way decreased equivalent dIN firing frequency ("half cycle" period is the start of mid-cycle IPSP to dIN spike peak) to $93.9 \pm 0.4 \%$ of the control ( $n=6 \mathrm{dINs}$ in 6 tadpoles, $p<0.001$, Wilcoxon signed rank test; Fig. $10 D, E)$. There is also a simultaneous decrease of swimming frequency (97.6 $\pm 3 \%$ of control, $p<0.001$; Fig. $10 F)$. For the swimming episodes where the IPSPs appeared depolarizing but did not evoke dIN firing (Fig. $10 \mathrm{D}$, dark blue traces), the drop in swimming frequency was slightly larger $(93 \pm 2.5 \%, p<0.001$; Fig. $10 F)$. Similar loading of high $\mathrm{Cl}^{-}$into other types of neurons resulted in no change in swimming frequency ( $n=4$, data not shown). Second, we injected short positive current pulses $(2-10 \mathrm{~ms},<500$ pA) time-locked to dIN spikes during swimming with $\sim 25 \mathrm{~ms}$ delay to weaken IPSPs (Fig. 10G,H). The frequency of dIN firing in the cycles with current injections $(22.11 \pm 0.21 \mathrm{~Hz})$ was significantly lower than that of the preceding cycle $(23.06 \pm 0.28 \mathrm{~Hz}$, 8 dINs, 5 pairs of cycles measured from each dIN, $p<0.0001$, paired $t$ test). The swimming frequency for the cycles with current injections $(22.7 \pm 0.32 \mathrm{~Hz})$, however, did not differ from that of the preceding cycle $(22.68 \pm 0.26 \mathrm{~Hz}, p>0.05)$.

\section{Discussion}

Rhythmic firing of neurons in the locomotor circuit is driven by oscillatory membrane potentials, i.e., alternating depolarization and hyperpolarization. Many factors that can help terminate the burst firing of neurons (Grillner et al., 1995) can affect rhythm frequency (e.g., calcium-dependent potassium currents) (Hellgren et al., 1992; el Manira et al., 1994). In this study, we investigated how excitation and inhibition can influence rhythm speed at the most basic level. It is known that locomotor speed is related to glutamatergic activation levels in the spinal cord (Brodin et al., 1985; Alford and Grillner, 1990; Cazalets et al., 1992; Brocard et al., 2010). Blocking NMDARs in neonatal rat spinal cord suppressed low-frequency motor rhythms (Beato et al., 1997; Cowley
Table 1. Effects of strychnine on neuronal resting properties and spikes

\begin{tabular}{lllll}
\hline & $\begin{array}{l}\text { Resting membrane } \\
\text { potential } \\
(\mathrm{mV})\end{array}$ & $\begin{array}{l}\text { Cellular input } \\
\text { resistance } \\
(\mathrm{M} \Omega)\end{array}$ & $\begin{array}{l}\text { Spike } \\
\text { height } \\
(\mathrm{mV})\end{array}$ & $\begin{array}{l}\text { Spike } \\
\text { width at } \\
0 \mathrm{mV}(\mathrm{ms})\end{array}$ \\
\hline Neurons & $\begin{array}{l}9 \mathrm{dINs}, 2 \text { alNs, } 1 \mathrm{cIN}, \\
3 \text { motoneurons }\end{array}$ & $6 \mathrm{dINs}$ & $6 \mathrm{dINs}$ & $6 \mathrm{dINs}$ \\
Control & $-53.7 \pm 1.7$ & $241 \pm 10.7$ & $68.3 \pm 1.7$ & $2.19 \pm 0.09$ \\
Strychnine & $-54.3 \pm 1.8$ & $240 \pm 11$ & $67.5 \pm 1.8$ & $2.15 \pm 0.18$ \\
\hline
\end{tabular}

et al., 2005). Studies from neonatal mice previously showed that AP5 did not block 5-HT-evoked rhythms (Nishimaru et al., 2000; Whelan et al., 2000). A recent study on neonatal mice found that NMDARs contributed to low-frequency locomotor-like rhythms but at higher frequencies, non-NMDAR activation was necessary (Talpalar and Kiehn, 2010). These fictive locomotor-like rhythmic episodes were usually induced by exogenous chemicals including NMDA, glutamate, or 5-HT, or by continuously stimulating the brainstem, which may not resemble the more natural episodes that are normally initiated by a brief sensory stimulus (Gabriel et al., 2011). In young Xenopus tadpoles, free mechanosensory nerve endings from Rohon-Beard neurons in the spinal cord have developed to innervate the whole tadpole trunk skin (Roberts et al., 2010). An advantage of using the tadpole preparation is that self-sustaining fictive swimming can be initiated by briefly stimulating the animal skin mechanically or electrically, avoiding the use of exogenous excitants. Importantly, the different types of neuron in the swimming circuit and their synaptic connection have been identified. Approximately 10 types of neuron have been found to be involved in tadpole swimming. Among them, dINs, cINs, aINs, and motoneurons are rhythmically active during swimming and are responsible for generating swimming rhythms (Fig. 11 A) (Roberts et al., 2010; Li 2011). 


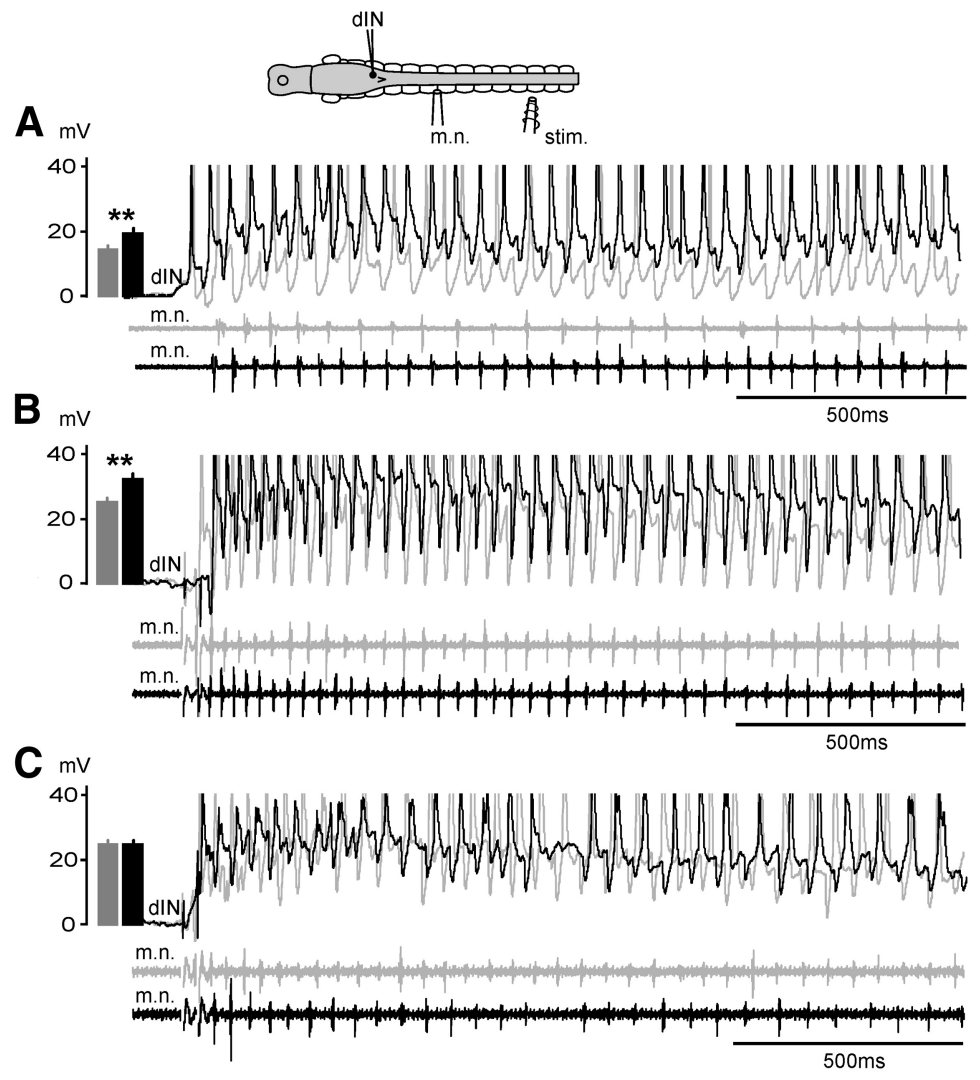

Figure 9. Strychnine but not extracellular $\mathrm{Cl}^{-}$substitution enhanced dIN background excitation during swimming. $\boldsymbol{A}, \mathrm{Mi}-$ croperfused $0.5 \mu \mathrm{m}$ strychnine partially blocks inhibition in a dIN while enhancing dIN background excitation in normal saline containing $1 \mathrm{~mm} \mathrm{Mg}^{2+}$. $\boldsymbol{B}$, Bath-applied $0.15 \mu \mathrm{m}$ strychnine enhances the background excitation in a dIN when $\mathrm{Mg}^{2+}$ is omitted from the saline. $C$, Saline with extracellular chloride lowered to $25 \mathrm{~mm}$ weakened inhibition without affecting dIN background excitation. $\boldsymbol{A}-\boldsymbol{C}$, Bar charts are averaged measurements of background excitation in the first second after swimming is started. **Significance levels at $p<0.01$ ( $t$ test). dIN records are overlapped for clarity. Control traces are gray. The resting membrane potential for records in $\boldsymbol{A}$ is $-52 \mathrm{mV}$, in $\boldsymbol{B}-53 \mathrm{mV}$, and in $\boldsymbol{C}-49 \mathrm{mV}$. Spikes are truncated. Note swimming frequency (m.n.) increases in $\boldsymbol{A}$ and $\boldsymbol{B}$ when strychnine is applied but drops in $\boldsymbol{C}$ when chloride is lowered.

We have recently identified that dINs drive tadpole swimming rhythms (Li et al., 2006, 2009, 2010; Soffe et al., 2009). All dINs have descending axons but approximately half of the rostral dIN population (in the caudal hindbrain and rostral spinal cord) also have ascending axons. Rostral dINs fire earliest during swimming and they form positive feedback connections via ascending axons to maintain stable excitation in themselves while their descending axons send rhythmic excitation to the spinal cord to drive the more caudal end of the swimming circuit (Fig. 11 A) (Li et al., 2006; Soffe et al., 2009). This means that swimming frequency is dictated by the rate at which these rostral dINs fire. This is different from the widely accepted notion that brainstem commands to spinal locomotor circuit are tonic and unpatterned, providing background excitation for the spinal circuit where the locomotor rhythm frequency is determined (for discussions, see Soffe et al., 2009). Therefore, understanding how dIN firing is controlled will provide direct insights into how tadpole swimming frequency is controlled.

We first analyzed what changes inward currents in dINs underwent during swimming and how the changes could determine dIN firing rates. Unlike other types of rhythmic neuron, dINs only fire a single spike at the onset of depolarizing current pulses. However, they can fire rebound spikes following phasic inhibition or repetitively via NMDAR-dependent pacemaker properties (Li et al., 2006, 2010). In this study, we found that, during swimming, tonic inward current/depolarization decreases when the swimming frequency drops. NMDAR activation from repetitive dIN firing was shown to be able to summate in tadpole motoneurons and produce background depolarization (Dale and Roberts, 1985). Similar summation of NMDAR excitation can take place in dINs. The NMDAR excitation can come from brief firing of sensory pathway interneurons following skin stimulation (Li et al., 2003). During swimming, dINs activate nicotinic, AMPA, and NMDA receptors simultaneously on all rhythmic neurons, including themselves ( $\mathrm{Li}$ et al., 2004a). The long-lasting NMDAR current can outlast several swimming cycles and contribute to background depolarization while nicotinic and AMPA receptors mediate phasic fast EPSCs during swimming. NMDAR currents from both sensory inputs and dIN activity will summate at the start of swimming but during swimming, dINs are the predominant sources for NMDAR excitation. This may explain the decreasing tonic inward currents seen within the first few seconds of swimming. Extensive electrical coupling among dINs may also contribute to background depolarization because the coupling coefficient is highest for low-frequency signals ( $\mathrm{Li}$ et al., 2009). In contrast, the size of phasic EPSCs in dINs remains largely unchanged when swimming frequency drops. This is consistent with the observation that dIN firing reliability is stable throughout swimming. It was shown previously that strong depolarization can promote faster NMDAR-dependent pacemaker firing in dINs (Li et al., 2010). We have shown in this study that strong background depolarization can also sustain faster dIN rebound firing. Faster dIN firing from both mechanisms can lead directly to faster swimming rhythms.

We then analyzed changes in phasic inhibition in dINs during swimming and how they could affect dIN rebound firing speed and swimming. cINs and aINs provide phasic inhibition, the amplitude of which decreases with falling swimming frequencies, onto dINs during swimming (Fig. 11A). Bigger aIN IPSCs at higher swimming frequencies fit with their higher firing reliability when swimming starts (Li et al., 2004b). Interestingly, though cIN firing remained stable, cIN IPSCs showed a steady decline with progressing swimming activity. This may imply that cIN transmission is more susceptible to some activity-dependent run-down mechanisms (Dale and Gilday, 1996). Previous studies on the role of inhibition in locomotor frequency control have yielded conflicting observations. Weakening inhibition led to faster swimming rhythms in lampreys by lesioning commissural axons (Cangiano and Grillner, 2003) and in tadpoles by applying low concentrations of strychnine (Dale, 1995). When phasic inhibition was strengthened by noradrenalin, the rhythms were slowed down in tadpoles (McDearmid et al., 1997). These studies suggest that inhibition might be weak when swimming frequency is high. However, previous studies in lamprey found that lower 
doses of strychnine (McPherson et al., 1994) or ablation of commissural inhibitory interneurons (Buchanan and McPherson, 1995) increased cycle periods of fictive swimming induced by NMDA. Genetically or acutely silencing local inhibitory interneurons, which expressed transcription factor Engrailed 1, also slowed down rhythmic motor bursting in neonatal mice (Gosgnach et al., 2006). Tadpole aINs express Engrailed 1 and we have proposed that one of their roles was to limit multiple firing in rhythmic neurons (Li et al., 2004b). Here we show that aIN inhibition can assist cIN inhibition to speed up dIN rebound firing when the latter is weak. This reveals a new role of aINs in regulating swimming frequencies. Blocking glycinergic aIN and cIN transmission during swimming was accompanied with enhanced background excitation. One plausible explanation is that following blockade of glycinergic receptors, the overall cellular input resistance in rhythmic neurons will increase during swimming. Similar tonic inward currents then can produce larger background depolarization. More specifically, reducing dIN membrane potential hyperpolarization via chloride replacement or timed current injections during swimming slowed down dIN rebound firing. This suggests that the role of phasic inhibition in swimming frequency control has two facets: speeding up rhythms directly by facilitating rebound firing and slowing down rhythms indirectly by shunting inward currents. The slowing down of locomotor-like rhythms following inhibitory interneuron ablation (Buchanan and McPherson, 1995; Gosgnach et al., 2006) may be caused by significant weakening of rebound mechanisms.

It is rare to be able to change the network activity by adjusting single interneuron activities in vertebrates (but see Houweling and Brecht, 2008). It has been occasionally possible to alter network activity by exciting individual interneurons in tadpoles ( $\mathrm{Li}$ et al., 2004c, 2006). It is likely that some dINs recorded in this paper are powerful individuals. Alternatively, we recently showed that dINs form extensive but almost exclusive electrical coupling among themselves (Li et al., 2009). Current injections or intracellular chloride loading into single dINs can affect multiple dINs by means of gap junctions. Either way, the experimental manipulations would be specific to the dIN population.

The roles of excitation and inhibition in network functions are often difficult to separate due to the interactions between ionotropic receptors (e.g., glutamatergic and glycinergic transmission) (Johnson and Ascher, 1987; Kleckner and Dingledine, 1988; Liu et $\mathrm{Mg}^{2+}$ in $\boldsymbol{D}-\boldsymbol{H}$.
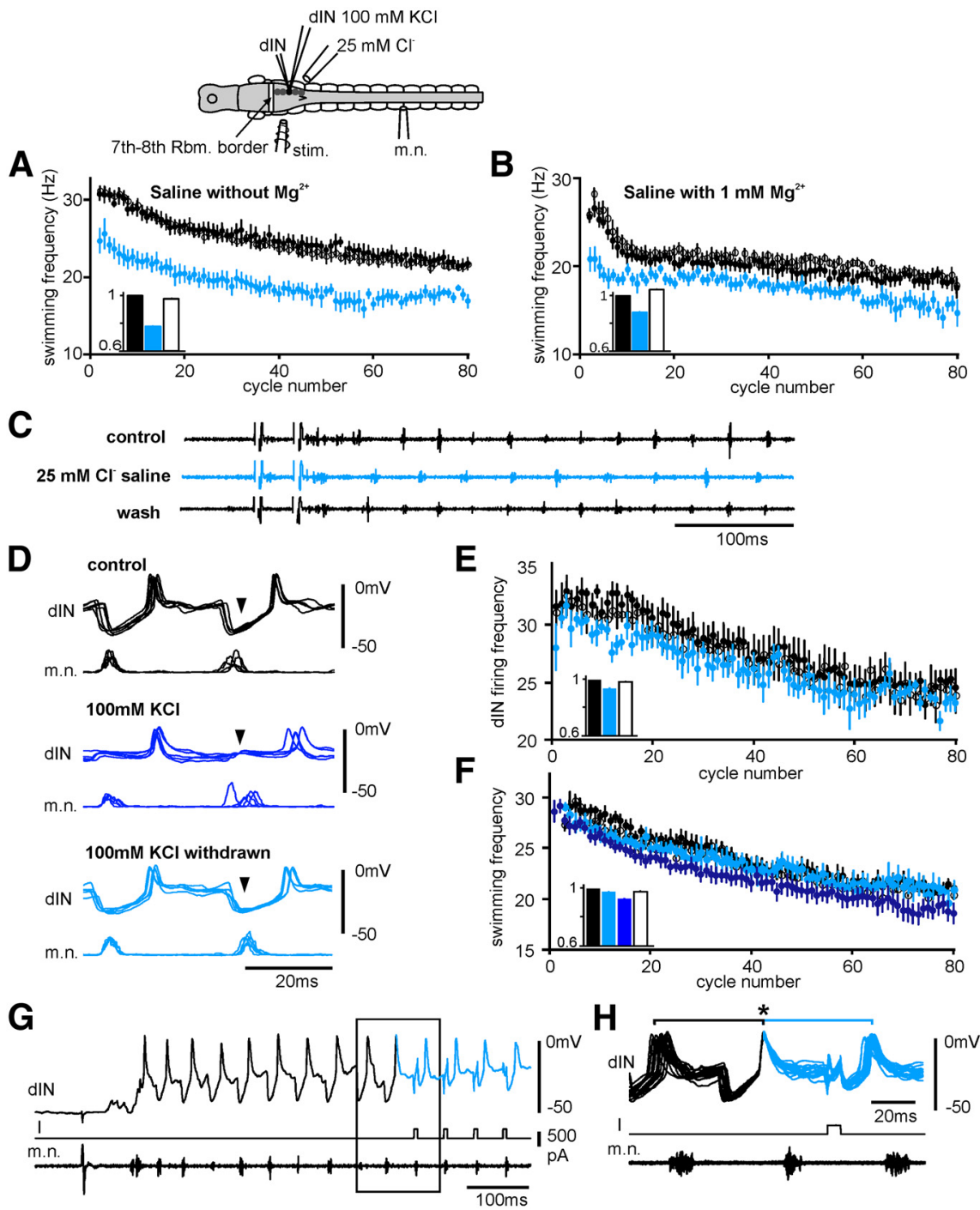

Figure 10. Weakening inhibition slows down dIN firing and swimming frequency. $\boldsymbol{A}$, Time series measurements of swimming frequencies with $\mathrm{Mg}^{2+}$ omitted from saline in control (filled circles), $25 \mathrm{~mm} \mathrm{Cl}^{-}$saline (blue), and wash (empty circles). $\boldsymbol{B}$, Similar measurements to those in $\boldsymbol{A}$ but saline contains $1 \mathrm{~mm} \mathrm{Mg}{ }^{2+}$. C, Example records of swimming slowed down by saline with $25 \mathrm{~mm}$ $\mathrm{Cl}^{-}$(blue) for data in $\boldsymbol{B} . \boldsymbol{A}, \boldsymbol{B}$, Bar charts are averaged swimming frequency measurements normalized to control in each cycle. Frequency changes compared with control are significant in all cases (Wilcoxon signed rank test, $p<0.01$ ). $\boldsymbol{D}$, Loading a dIN with $100 \mathrm{~mm} \mathrm{KCl}$ using a second whole-cell recording electrode (traces not shown, see top diagram) weakens IPSPs (arrowheads) in the dIN during swimming. Overlapped traces are lined to rectified motor nerve bursts (m.n., 5 in each case). IPSPs appear depolarizing when $100 \mathrm{~mm} \mathrm{KCl}$ electrode is not withdrawn (dark blue) and are smaller than control when the electrode is moved slightly away from the soma (light blue). $\boldsymbol{E}$, Time series measurements of equivalent dIN rebound firing frequency in control (filled circles), high $\mathrm{KCl}$ recording (light blue), and withdrawal of high $\mathrm{KCl}$ electrode (empty circles). Period from the start of midcycle IPSP to dIN spike peak (half a cycle) is used for calculating equivalent dIN rebound firing frequency. $\boldsymbol{F}$, Similar time series to $\boldsymbol{E}$ but measurements are for m.n. bursts. Dark blue symbols are episodes when IPSPs are depolarizing but not evoking dIN spikes (D, dark blue traces). $\boldsymbol{G}$, An example record when short positive current pulses (I) are injected at the beginning of inhibition (blue) during swimming. Box marks the two cycles used for comparing dIN firing frequency. $\boldsymbol{H}$, Overlapped sweeps to show current injection time-locked to dIN spikes slows down dIN firing $\left({ }^{*}, n=18\right.$ trials in this recording, $21.5 \pm 0.3 \mathrm{~Hz}$ in control to $20.7 \pm 0.1 \mathrm{~Hz}, p<0.05$, paired $t$ test) while the swimming frequency is not affected (control: $21 \pm 0.1 \mathrm{~Hz}$, current injection: $20.7 \pm 0.2 \mathrm{~Hz}$ ). Saline does not contain

al., 2010) and the existence of voltage-dependent ion channels (e.g., rebound properties) (Getting, 1989; Harris-Warrick, 2002; Llinás, 1988; Perez-Reyes, 2003). As we show here in a model locomotor circuit, both strong background depolarization and strong phasic inhibition are present in fast swimming rhythms. Similar positive correlation between synaptic current and locomotion frequency has recently been observed in adult zebrafish motoneurons during fictive swimming (Gabriel et al., 2011). We 


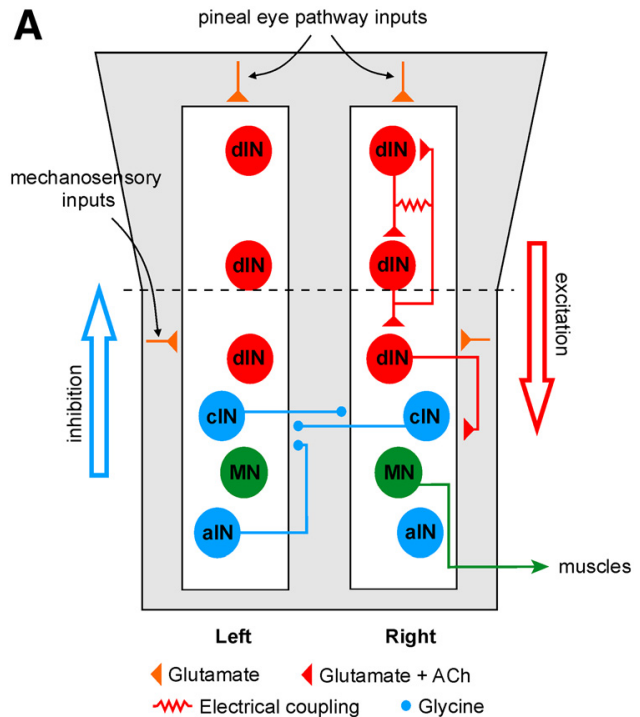

B

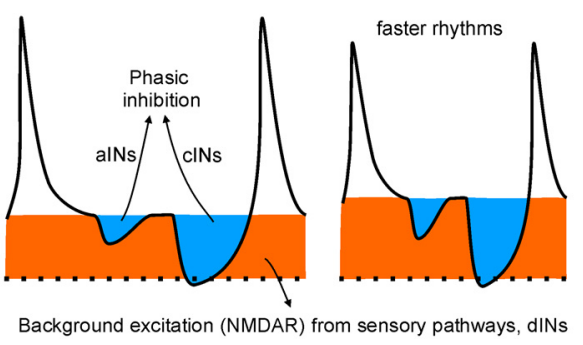

Figure 11. Tadpole swimming circuit and a working model for swimming frequency control. $A$, The swimming rhythm generating circuit comprises symmetrical left and right half centers that are located in the spinal cord and caudal hindbrain and contain four types of neuron [dIN, $\mathrm{CIN}$, alN, and motoneuron (MN)]. The swimming rhythms are generated in the dINs in the caudal hindbrain and rostral spinal cord region and rhythmic excitation (red arrow) propagates and is relayed to the spinal cord by dIN descending axons. The ascending axons of rostral dINs form feedback excitation connections in the caudal hindbrain and rostral spinal cord. Phasic inhibition (blue arrow) arises mostly from the cINs and alNs in the spinal cord. Dashed line indicates the anatomical border between the spinal cord (below) and hindbrain (above). Synapses on boxes mean all neurons within are innervated. $\boldsymbol{B}$, Left, Representation of dIN activity during one swimming cycle highlighting the factors that influence swimming frequencies. Phasic inhibition and background excitation levels are colored blue and orange, respectively. Dotted line marks resting membrane potential. Right, High-frequency swimming is correlated with strong background excitation and phasic inhibition.

conclude that both strong excitation and inhibition can promote fast network rhythms. Where rebound properties play a role in patterning neuronal activities like in the locomotor circuit, the strengths of excitation and inhibition may be tuned together rather than separately (Fig. $11 B$ ).

\section{References}

Alford S, Grillner S (1990) CNQX and DNQX block non-NMDA synaptic transmission but not NMDA-evoked locomotion in lamprey spinal cord. Brain Res 506:297-302.

Alonso A, Llinás RR (1989) Subthreshold $\mathrm{Na}^{+}$-dependent theta-like rhythmicity in stellate cells of entorhinal cortex layer II. Nature 342:175-177.

Beato M, Bracci E, Nistri A (1997) Contribution of NMDA and non-NMDA glutamate receptors to locomotor pattern generation in the neonatal rat spinal cord. Proc Biol Sci 264:877-884.

Bhatt DH, McLean DL, Hale ME, Fetcho JR (2007) Grading movement strength by changes in firing intensity versus recruitment of spinal interneurons. Neuron 53:91-102.

Brocard F, Tazerart S, Vinay L (2010) Do pacemakers drive the central pattern generator for locomotion in mammals? Neuroscientist 16:139-155.

Brodin L, Grillner S, Rovainen CM (1985) N-methyl-D-aspartate (NMDA), kainate and quisqualate receptors and the generation of fictive locomotion in the lamprey spinal cord. Brain Res 325:302-306.

Buchanan JT, McPherson DR (1995) The neuronal network for locomotion in the lamprey spinal cord: evidence for the involvement of commissural interneurons. J Physiol Paris 89:221-233.

Buzsáki G (2002) Theta oscillations in the hippocampus. Neuron 33:325-340.

Cangiano L, Grillner S (2003) Fast and slow locomotor burst generation in the hemispinal cord of the lamprey. J Neurophysiol 89:2931-2942.

Cazalets JR, Sqalli-Houssaini Y, Clarac F (1992) Activation of the central pattern generators for locomotion by serotonin and excitatory amino acids in neonatal rat. J Physiol 455:187-204.

Cowley KC, Zaporozhets E, Maclean JN, Schmidt BJ (2005) Is NMDA receptor activation essential for the production of locomotor-like activity in the neonatal rat spinal cord? J Neurophysiol 94:3805-3814.

Crone SA, Zhong G, Harris-Warrick R, Sharma K (2009) In mice lacking V2a interneurons, gait depends on speed of locomotion. J Neurosci 29:7098-7109.

Dale N (1995) Experimentally derived model for the locomotor pattern generator in the Xenopus embryo. J Physiol 489:489-510.

Dale N, Gilday D (1996) Regulation of rhythmic movements by purinergic neurotransmitters in frog embryos. Nature 383:259-263.

Dale N, Roberts A (1985) Dual component amino-acid-mediated synaptic potentials: excitatory drive for swimming in Xenopus embryos. J Physiol 363:35-59.

el Manira A, Tegnér J, Grillner S (1994) Calcium-dependent potassium channels play a critical role for burst termination in the locomotor network in lamprey. J Neurophysiol 72:1852-1861.

Feldman JL, Del Negro CA (2006) Looking for inspiration: new perspectives on respiratory rhythm. Nat Rev Neurosci 7:232-242.

Fontanini A, Bower JM (2006) Slow-waves in the olfactory system: an olfactory perspective on cortical rhythms. Trends Neurosci 29:429-437.

Gabriel JP, Ausborn J, Ampatzis K, Mahmood R, Eklöf-Ljunggren E, El Manira A (2011) Principles governing recruitment of motoneurons during swimming in zebrafish. Nat Neurosci 14:93-99.

Getting PA (1989) Emerging principles governing the operation of neural networks. Annu Rev Neurosci 12:185-204.

Gosgnach S, Lanuza GM, Butt SJ, Saueressig H, Zhang Y, Velasquez T, Riethmacher D, Callaway EM, Kiehn O, Goulding M (2006) V1 spinal neurons regulate the speed of vertebrate locomotor outputs. Nature 440 : 215-219.

Grillner S, Jessell TM (2009) Measured motion: searching for simplicity in spinal locomotor networks. Curr Opin Neurobiol 19:572-586.

Grillner S, Deliagina T, Ekeberg O, el Manira A, Hill RH, Lansner A, Orlovsky GN, Wallén P (1995) Neural networks that co-ordinate locomotion and body orientation in lamprey. Trends Neurosci 18:270-279.

Harris-Warrick RM (2002) Voltage-sensitive ion channels in rhythmic motor systems. Curr Opin Neurobiol 12:646-651.

Hellgren J, Grillner S, Lansner A (1992) Computer simulation of the segmental neural network generating locomotion in lamprey by using populations of network interneurons. Biol Cybern 68:1-13.

Houweling AR, Brecht M (2008) Behavioural report of single neuron stimulation in somatosensory cortex. Nature 451:65-68.

Johnson JW, Ascher P (1987) Glycine potentiates the NMDA response in cultured mouse brain neurons. Nature 325:529-531.

Kleckner NW, Dingledine R (1988) Requirement for glycine in activation of NMDA-receptors expressed in Xenopus oocytes. Science 241:835-837.

Li WC (2011) Generation of locomotion rhythms without inhibition in vertebrates: the search for pacemaker neurons. Integr Comp Biol 51:879-889.

Li WC, Soffe SR, Roberts A (2003) The spinal interneurons and properties of glutamatergic synapses in a primitive vertebrate cutaneous flexion reflex. J Neurosci 23:9068-9077.

Li WC, Soffe SR, Roberts A (2004a) Glutamate and acetylcholine corelease at developing synapses. Proc Natl Acad Sci U S A 101:15488-15493.

Li WC, Higashijima S, Parry DM, Roberts A, Soffe SR (2004b) Primitive roles for inhibitory interneurons in developing frog spinal cord. J Neurosci 24:5840-5848.

Li WC, Soffe SR, Roberts A (2004c) Dorsal spinal interneurons forming a primitive, cutaneous sensory pathway. J Neurophysiol 92:895-904.

Li WC, Soffe SR, Wolf E, Roberts A (2006) Persistent responses to brief 
stimuli: feedback excitation among brainstem neurons. J Neurosci 26:4026-4035.

Li WC, Roberts A, Soffe SR (2009) Locomotor rhythm maintenance: electrical coupling among premotor excitatory interneurons in the brainstem and spinal cord of young Xenopus tadpoles. J Physiol 587:1677-1693.

Li WC, Roberts A, Soffe SR (2010) Specific brainstem neurons switch each other into pacemaker mode to drive movement by activating NMDA receptors. J Neurosci 30:16609-16620.

Liu J, Wu DC, Wang YT (2010) Allosteric potentiation of glycine receptor chloride currents by glutamate. Nat Neurosci 13:1225-1232.

Llinás RR (1988) The intrinsic electrophysiological properties of mammalian neurons: insights into central nervous system function. Science 242:1654-1664.

Mann EO, Paulsen O (2005) Mechanisms underlying gamma ('40 Hz') network oscillations in the hippocampus: a mini-review. Prog Biophys Mol Biol 87:67-76.

Mayer ML, Westbrook GL, Guthrie PB (1984) Voltage-dependent block by $\mathrm{Mg}^{2+}$ of NMDA responses in spinal cord neurones. Nature 309:261-263.

McDearmid JR, Scrymgeour-Wedderburn JF, Sillar KT (1997) Aminergic modulation of glycine release in a spinal network controlling swimming in Xenopus laevis. J Physiol 503:111-117.

McLean DL, Fan J, Higashijima S, Hale ME, Fetcho JR (2007) A topographic map of recruitment in spinal cord. Nature 446:71-75.

McLean DL, Masino MA, Koh IY, Lindquist WB, Fetcho JR (2008) Continuous shifts in the active set of spinal interneurons during changes in locomotor speed. Nat Neurosci 11:1419-1429.

McPherson DR, Buchanan JT, Kasicki S (1994) Effects of strychnine on fictive swimming in the lamprey: evidence for glycinergic inhibition, discrepancies with model predictions, and novel modulatory rhythms. J Comp Physiol A 175:311-321.
Nishimaru H, Takizawa H, Kudo N (2000) 5-hydroxytryptamine-induced locomotor rhythm in the neonatal mouse spinal cord in vitro. Neurosci Lett 280:187-190.

Perez-Reyes E (2003) Molecular physiology of low-voltage-activated t-type calcium channels. Physiol Rev 83:117-161.

Perrins R, Walford A, Roberts A (2002) Sensory activation and role of inhibitory reticulospinal neurons that stop swimming in hatchling frog tadpoles. J Neurosci 22:4229-4240.

Rivlin-Etzion M, Marmor O, Heimer G, Raz A, Nini A, Bergman H (2006) Basal ganglia oscillations and pathophysiology of movement disorders. Curr Opin Neurobiol 16:629-637.

Roberts A (1978) Pineal eye and behaviour in Xenopus tadpoles. Nature 273:774-775.

Roberts A, Li WC, Soffe SR (2010) How neurons generate behavior in a hatchling amphibian tadpole: an outline. Front Behav Neurosci 4:16.

Soffe SR (1991) Triggering and gating of motor responses by sensory stimulation: behavioural selection in Xenopus embryos. Proc Biol Sci 246:197-203.

Soffe SR, Roberts A, Li WC (2009) Defining the excitatory neurons that drive the locomotor rhythm in a simple vertebrate: insights into the origin of reticulospinal control. J Physiol 587:4829-4844.

Steriade M (2005) Sleep, epilepsy and thalamic reticular inhibitory neurons. Trends Neurosci 28:317-324.

Talpalar AE, Kiehn O (2010) Glutamatergic mechanisms for speed control and network operation in the rodent locomotor CpG. Front Neural Circuits 4.pii:19.

Whelan P, Bonnot A, O’Donovan MJ (2000) Properties of rhythmic activity generated by the isolated spinal cord of the neonatal mouse. J Neurophysiol 84:2821-2833. 